\title{
LPS-Is It a Major Liability Factor for Cancer Risk and Severity?
}

\author{
Muthuswamy Balasubramanyam* \\ Cell and Molecular Biology, Madras Diabetes Research Foundation (MDRF), ICMR-Centre for Advanced Research \\ on Diabetes, Gopalapuram, Chennai 600086, India
}

\begin{abstract}
Lipopolysaccharide (LPS) is a major integral component of the outer membrane of Gram-negative bacteria cell walls, that is released when bacteria lyse. LPS was shown to stimulate immune system cells by binding the cell-surface Toll-like receptor 4 (TLR4) and activating transcription factors and protein kinases, such as NF-kB and p38 kinase. This in turn was shown to result in an increased production of proinflammatory cytokines and overexpression of cell adhesion molecules and matrix-degrading enzymes. ${ }^{1}$ The pathogenic role of LPS has been shown to be linked to several disease-states, viz., liver diseases, neurological degeneration, chronic inflammation of the gut, diabetes, and prostate cancer metastasis. ${ }^{2-4}$ One of our pioneering works ${ }^{5}$ showed increased circulatory levels of LPS and Zonulin as novel biomarkers of proinflammation in patients with type 2 diabetes. LPS is also proatherogenic factor and apart from its increase in blood levels in patients with atherosclerosis, LPS from Escherichia coli has also been shown to localize in carotid plaques, implying a mediatory role in atherosclerotic lesions. ${ }^{6}$
\end{abstract}

\section{Is There an Association of Circulatory LPS and Risk of Ad- vanced Colorectal Adenoma (ACA)?}

In a recent study, Chen et al. ${ }^{7}$ investigated the association between LPS serum levels and risk of ACA.In that colonoscopy clinic-based study, male patients with diagnosis of ACA and polyp-free control individuals were phenotyped for comparative analyses with age, ethnicity, and information on demographics, lifestyle, and medical history along with serum levels of LPS measurement.Examining the LPS levels based on the characteristics and substratification of adenoma, the investigators showed that patients with largesized adenoma $(>2 \mathrm{~cm})$, villous adenoma, or high-grade dysplasia tended to have higher serum levels of LPS compared to patients with smaller, tubular adenoma, and adenoma without high-grade dysplasia, respectively. Although LPS levels were slightly higher in patients with ACA compared to controls, the subtle differences in the LPS levels did not reach statistical significance. With the results from multiple regression analysis, the authors concluded that there was no statistically significant association between LPS and

\footnotetext{
Abbreviations: ACA, advanced colorectal adenoma; CRC, colorectal cancer; IAP, intestinal alkaline phosphatase; LAL, Limulus amoebocyte lysate; LBP, LPS-binding protein; LPS, Lipopolysaccharide; TLR-4, Toll-like receptor 4

Received: June 02, 2020; Revised: June 05, 2020; Accepted: June 05, 2020

*Correspondence to: Muthuswamy Balasubramanyam, Department of Cell and Molecular Biology, Madras Diabetes Research Foundation, ICMR-Centre for Advanced Research on Diabetes, Gopalapuram, Chennai 600086, India. Tel: 9144-2835-9048, Fax:9144-2835-0935, E-mail: baluglobaldiab@gmail.com, balusignal@gmail.com How to cite this article: Balasubramanyam M. LPS - Is It a Major Liability Factor for Cancer Risk and Severity? Exploratory Research and Hypothesis in Medicine 2020;5(2):39-40. doi: 10.14218/ERHM.2020.00037.
}

the risk of ACA. As both the groups in this study were equally confounded by obesity, diabetes and hypertension (conditions known to exhibit elevated levels of LPS), this finding could have been attributed to the lack of statistical significance in the LPS levels. However, authors have admitted that larger future studies are warranted to further investigate the association between the systemic levels of LPS and risk of colorectal tumors, and to gain a better understanding of the relevant clinical implications.

\section{The Biological Link Between Gut Microbiota and Cancer Risk Attests to a Role for LPS}

Recent studies imply that gut dysbiosis and leakage of bacterial components (majorly LPS), may contribute to the metabolic disturbances and systemic inflammation linked to several diseasestates. More importantly, the gut microbiota has emerged as a central player that mechanistically links various risk factors to colorectal cancer (CRC) pathogenesis. ${ }^{8,9}$ While increased intestinal permeability is strongly linked to elevated level of gut microbiota-associated LPS-induced local inflammation, both of these phenomena were shown independently associated with CRC.10,11 In a multicohort CRC metagenome study, it was shown that the composition of specific bacteria enrichened in CRC patients were also correlated to LPS-related pathway. ${ }^{12}$ These studies attest to a definite role of LPS as a mediator in the genesis of CRC and its progression to severity.

\section{The Need for a Panel of Biomarkers to Assess CRC Risk}

The Limulus amoebocyte lysate (LAL) test, an assay that is routinely used to quantify LPS as an endotoxin, has limitations due to the levels of LPS in the systemic circulation of healthy humans and within those with various clinical disorders, so that they can vary over a wide range. ${ }^{13}$ Because of its short half-life, low concentration, and high susceptibility to interfering substances, such as endogenous inhibitors of LPS, the utility of LPS detection via the LAL method has limitation in the routine clinical setting and large-scale studies. ${ }^{14}$ This definitely warrants the need for development of more reliable methods for LPS detection. In order to communicate with the innate immune system, LPS binds to the LPSbinding protein (LBP), which is pivotal for the binding of CD14 and transfer to the TLR4 complex. ${ }^{15}$ Therefore, LBP measurement is considered as a surrogate marker of metabolic endotoxemia. Indeed, an increased LBP level has been shown to be closely associated with obesity, prediabetes, and atherosclerosis, ${ }^{16-18}$ and has been observed as a significant and independent predictor of coronary artery disease. ${ }^{19}$ Moreover, LBP and CD14 polymorphisms 
have been shown to be correlated with increased CRC risk in Han Chinese. ${ }^{20}$ While intestinal alkaline phosphatase (IAP) has now emerged as a new factor that is essential for maintaining proper gut homeostasis, the role of IAP in detoxifying LPS has been demonstrated in several studies. ${ }^{21,22}$

Considering the above, it is important that studies that profile the biomarker role of circulatory levels of LPS in relation to disease-states should consider the LPS pathway approach and collectively measure a panel of circulatory biomarkers, viz., LPS, LBP, soluble CD14, Zonulin, and IAP. These potential biomarkers have to be correlated to conventional risk factors of $\mathrm{CRC}$, so as to make further improvement(s) in disease prediction, treatment and management. In this context, a recent study by Yin et al. ${ }^{23}$ used multiomics-based prognostic analysis and target-regulation simulation modeling and detected several prognosis risk biomarkers for colon adenocarcinoma (which includes LBP). Therefore, it is advised that an LPS pathway approach (rather than just measuring circulatory LPS levels) would be the ideal way not only to predict the disease-risk but also to unravel new perspectives for novel drug development and therapeutic applications for colorectal adenoma as well as other non-communicable diseases.

\section{Acknowledgments}

None.

Funding

None.

\section{Conflict of interest}

The author has no conflict of interests related to this publication.

\section{References}

[1] Manthey CL, Brandes ME, Perera PY, Vogel SN. Taxol increases steady-state levels of lipopolysaccharide-inducible genes and protein tyrosine phosphorylation in murine macrophages. J Immunol 1992;149(7):2459-2465.

[2] Magdaleno F, Blajszczak CC, Nieto N. Key events participating in the pathogenesis of alcoholic liver disease. Biomolecules 2017;7(1):9. doi:10.3390/biom7010009.

[3] Gomes JMG, Costa JA, Alfenas RCG. Metabolic endotoxemia and diabetes mellitus: A systematic review. Metabolism 2017;68:133-144. doi:10.1016/j.metabol.2016.12.009.

[4] Jain S, Dash P, Minz AP, Satpathi S, Samal AG, Behera PK, et al. Lipopolysaccharide (LPS) enhances prostate cancer metastasis potentially through NF-KB activation and recurrent dexamethasone administration fails to suppress it in vivo. Prostate 2019;79(2):168-182. doi:10.1002/pros.23722.

[5] Jayashree B, Bibin YS, Prabu D, Shanthirani CS, Gokulakrishnan K, Lakshmi BS, et al. Increased circulatory levels of Lipopolysaccharide (LPS) and Zonulin signify novel biomarkers of proinflammation in patients with type 2 diabetes. Mol Cell Biochem 2014;388(1-2):203210. doi:10.1007/s11010-013-1911-4.

[6] Carnevale R, Nocella C, Petrozza V, Cammisotto V, Pacini L, Sorrentino $\mathrm{V}$, et al. Localization of Lipopolysaccharide from Escherichia Coli into Human Atherosclerotic Plaque. Sci Rep 2018;8(1):3598. doi:10.1038/ s41598-018-22076-4.

[7] Chen E, Kalavari A, Bui-Thanh NA, Opekun AR, White DL, Rosen D, et al. Serum Levels of Lipopolysaccharides and Risk of Advanced Colorectal Adenoma. Explor Res Hypothesis Med 2020. doi:10.14218/ ERHM.2020.00001.

[8] Zitvogel L, Galluzzi L, Viaud S, Vétizou M, Daillère R, Merad M, et al. Cancer and the gut microbiota: an unexpected link. Sci Transl Med 2015;7(271):271ps1. doi:10.1126/scitransImed.3010473.

[9] Maisonneuve C, Irrazabal T, Martin A, Giardin SE, Philpott DJ. The Impact of the gut microbiome on colorectal cancer. Annu Rev Cancer Biol 2018;2:229-249. doi:10.1146/annurev-cancerbio-030617-050240.

[10] Soler AP, Miller RD, Laughlin KV, Carp NZ, Klurfeld DM, Mullin JM. Increased tight junctional permeability is associated with the development of colon cancer. Carcinogenesis 1999;20(8):1425-1431. doi:10.1093/carcin/20.8.1425.

[11] Zhu G, Huang Q, Huang Y, Zheng W, Hua J, Yang S, et al. Lipopolysaccharide increases the release of VEGF-C that enhances cell motility and promotes lymphangiogenesis and lymphatic metastasis through the TLR4-NF-KB/JNK pathways in colorectal cancer. Oncotarget 2016;7(45):73711-73724. doi:10.18632/oncotarget.12449.

[12] Dai Z, Coker OO, Nakatsu G, Wu WKK, Zhao L, Chen Z, et al. Multicohort analysis of colorectal cancer metagenome identified altered bacteria across populations and universal bacterial markers. Microbiome 2018;6(1):70. doi:10.1186/s40168-018-0451-2.

[13] Gnauck A, Lentle RG, Kruger MC. Chasing a ghost?-Issues with the determination of circulating levels of endotoxin in human blood. Crit Rev Clin Lab Sci 2016;53(3):197-215. doi:10.3109/10408363.2015.1 123215.

[14] Novitsky TJ. Limitations of the Limulus Amebocyte Lysate Test in Demonstrating Circulating Lipopolysaccharides. Ann N Y Acad Sci 1998;851:416-421. doi:10.1111/j.1749-6632.1998.tb09018.x.

[15] Hailman E, Lichenstein HS, Wurfel MM, Miller DS, Johnson DA, Kelley $\mathrm{M}$, et al. Lipopolysaccharide (LPS)-binding protein accelerates the binding of LPS to CD14. J Exp Med 1994;179(1):269-277. doi:10.1084/jem.179.1.269.

[16] Moreno-Navarrete JM, Ortega F, Serino M, Luche E, Waget A, Pardo G, et al. Circulating Lipopolysaccharide-Binding Protein (LBP) as a Marker of Obesity-Related Insulin Resistance. Int J Obes (Lond) 2012;36(11):1442-1449. doi:10.1038/ijo.2011.256.

[17] Serrano M, Moreno-Navarrete JM, Puig J, Moreno M, Guerra E, Ortega F, et al. Serum Lipopolysaccharide-Binding Protein as a Marker of Atherosclerosis. Atherosclerosis 2013;230(2):223-227. doi:10.1016/j.atherosclerosis.2013.07.004.

[18] Tilves CM, Zmuda JM, Kuipers AL, Nestlerode CS, Evans RW, Bunker $\mathrm{CH}$, et al. Association of Lipopolysaccharide-Binding Protein With Aging-Related Adiposity Change and Prediabetes Among African Ancestry Men. Diabetes Care 2016;39(3):385-391. doi:10.2337/dc151777.

[19] Lepper PM, Schumann C, Triantafilou K, Rasche FM, Schuster T, Frank $\mathrm{H}$, et al. Association of Lipopolysaccharide-Binding Protein and Coronary Artery Disease in Men. J Am Coll Cardiol 2007;50(1):25-31. doi:10.1016/j.jacc.2007.02.070.

[20] Chen R, Luo FK, Wang YL, Tang JL, Liu YS. LBP and CD14 Polymorphisms Correlate With Increased Colorectal Carcinoma Risk in Han Chinese. World J Gastroenterol 2011;17(18):2326-2331. doi:10.3748/wjg. v17.i18.2326.

[21] Goldberg RF, Austen WG Jr, Zhang X, Munene G, Mostafa G, Biswas $\mathrm{S}$, et al. Intestinal alkaline phosphatase is a gut mucosal defense factor maintained by enteral nutrition. PNAS 2008;105(9):3551-3556. doi:10.1073/pnas.0712140105.

[22] Bates JM, Akerlund J, Mittge E, Guillemin K. Intestinal alkaline phosphatase detoxifies lipopolysaccharide and prevents inflammation in zebrafish in response to the gut microbiota. Cell Host Microbe 2007;2(6):371-382. doi:10.1016/j.chom.2007.10.010.

[23] Yin Z, Yan X, Wang Q, Deng Z, Tang K, Cao Z, et al. Detecting Prognosis Risk Biomarkers for Colon Cancer Through Multi-Omics-Based Prognostic Analysis and Target Regulation Simulation Modeling. Front Genet 2020;doi:10.3389/fgene.2020.00524. 